\title{
LS Interference Alignment Algorithm Based on Symbol Detection Assistance
}

\author{
Guoqing Jia ${ }^{1}$, Junjun Du ${ }^{1}$, Xuebin Zheng ${ }^{2}$ \\ ${ }^{1}$ College of Physics and Electronic Information, Qinghai Nationalities University, Xining, China \\ ${ }^{2}$ Mechanical and Electrical Engineering, Hebei Normal University of Science \& Technology, Qinghuangdao, China \\ Email address: \\ guoqing.jia@qhmu.edu.cn(Guoqing Jia),729121938@qq.com (Junjun Du),1515982273@qq.com (Xuebin Zheng) \\ To cite this article: \\ Guoqing Jia, Junjun Du, Xuebin Zheng. LS Interference Alignment Algorithm Based on Symbol Detection Assistance. Mathematics and \\ Computer Science. Vol. 4, No. 1, 2019, pp. 1-5. doi: 10.11648/j.mcs.20190401.11
}

Received: December 23, 2018; Accepted: January 14, 2019; Published: April 18, 2019

\begin{abstract}
With the rapid growth of network users, how to increase the system capacity has become an urgent problem for the current communication system in the case of limited spectrum resources. The introduction of multi-user systems has increased system capacity, but it has also led to inter-user interference, which has further affected system capacity. To solve the multi-user interference problem, interference alignment is introduced. Interference Alignment (IA) is an interference cancellation technique that effectively eliminates the effects of interfering signals by compressing the interfering signal into a space independent of the desired signal and then forcing the interfering signal to zero at the receiving end. However, in practical applications, interference-aligned transceivers require a joint design, which is often difficult to achieve. The traditional approach is to mathematically expect it, but it also leads to some degree of irrationality in the transceiver design. In this paper, based on the traditional least square interference alignment (LS-IA) algorithm, a symbol-detection-assisted least square interference alignment (SDA-LS-IA) algorithm is proposed for its shortcomings in transceiver algorithm design. Firstly, based on the precoding matrix and the zero-forcing matrix of the transceiver designed by the traditional LS-IA, the symbol detection is performed, and then the transceiver is designed again according to the detection symbol, and then the symbol detection is performed. The computer simulation proves that the proposed algorithm has better anti-interference performance than the traditional LS-IA.
\end{abstract}

Keywords: Interference Alignment (IA), Interference Cancellation, Least Squares (LS), Symbol Detection Assistance

\section{Introduction}

With the development of the global mobile market, the popularity of smart terminals and the development of the Internet of Things business, mobile data traffic has grown rapidly [1-3]. In response to this situation, interference alignment was first proposed by S. Jafar [4]. The main idea of IA is to precode the signal before it is sent, and then perform zero-forcing filtering after receiving the signal to separate the signal into two separate spaces, thus eliminating interference. It shows that the theoretical maximum degree of freedom of the $K$ user interference channel can reach $K / 2$.

Suh $\mathrm{C}$ and Tse D firstly apply interference alignment to the cellular network and improve system freedom (DoF) by aligning the interfering signal with the subspace of the received signal [5]. An one-time linear precoding method is proposed on a single time slot frequency to achieve optimal degrees of freedom [6]. But it requires a joint design of the transmitter and receiver, which is often difficult to implement in practice. At the same time, in non-ideal channel, complete freedom cannot be achieved. Aiming at this problem, based on different optimization criteria (eg, minimum interference to interference leakage (MIL), maximum signal to interference plus noise ratio (Max-SINR), least squares (LS) minimum mean square (MMSE)), a number of iterative transceivers have been proposed [7-8].

The traditional LS-IA algorithm directly eliminates the influence of the data stream due to the use of mathematical expectation, resulting in the design of the transceiver is not strictly reasonable, especially in the case of insufficient data flow. Based on the traditional LS-IA, this paper proposes a symbol-detection-assisted least square interference alignment (SDA-LS-IA) algorithm, which is a LS-based transceiver design 
and symbolic detection joint iterative calculation algorithm. The proposed SDA-LS-IA algorithm has better performance than the traditional LS-IA by computer simulation.

\section{Interference System Model for LS Detection}

As shown in Figure 1, it is assumed that there are $2 K$ nodes of symmetric users in a multiple-input multiple-output (MIMO) multi-user interference system [9-12], where $K$ is the transmitter and the other $K$ nodes are receivers. In a wireless interference channel, each transmitter attempts to communicate only with the desired single receiver. Each transmitter carries $N$ transmitting antennas, each receiver with $M$ receiving antennas, and the transmitter communicates with the desired receiver by transmitting $d$ independent data streams.

Then the data received by the $i$ receiver can be expressed as:

$$
\boldsymbol{y}_{i}=\boldsymbol{H}_{i, j} \boldsymbol{V}_{i} \boldsymbol{s}_{i}+\sum_{j=1, j \neq i}^{K} \boldsymbol{H}_{i, j} \boldsymbol{V}_{j} \boldsymbol{s}_{j}+\boldsymbol{n}_{i}
$$

$\boldsymbol{y}_{i} \in \mathrm{C}^{M \times 1}$ represents the signal received by the $i$ node, $\mathrm{C}^{M \times 1}$ represents the complex matrix of dimension $M \times 1, \boldsymbol{H}_{i, j} \in \mathrm{C}^{M \times N}$ represents the channel matrix of the receiver transmitted from the transmitter of the $j$ node to the $i$ node, and $\boldsymbol{n}_{i} \in \mathrm{C}^{M \times 1}$ represents the channel noise of the $i$ node . Here, the model is constructed as an independent and identically distributed Gaussian matrix of zero mean and unit variance, that is, $\operatorname{vec}\left(\boldsymbol{H}_{i, j}\right) \sim \mathrm{N}_{\mathrm{C}}(\boldsymbol{\theta}, \boldsymbol{I}), \mathrm{N}_{\mathrm{C}}(\boldsymbol{\theta}, \boldsymbol{I})$ represents a complex matrix with a mean of 0 and a variance of $\boldsymbol{I}$, and $\boldsymbol{I}$ denotes an unit matrix. $\boldsymbol{V}_{j} \in \mathrm{C}^{N \times d}$ is the transmission precoding matrix on the $j$ transmitter, and $s_{j} \in \mathrm{C}^{d \times 1}$ is the $j$ transmitter transmission signal, which satisfies the condition $\mathrm{E}\left(\boldsymbol{s}_{j} \boldsymbol{s}_{j}^{\mathrm{H}}\right)=P \boldsymbol{I}, P$ represents power.

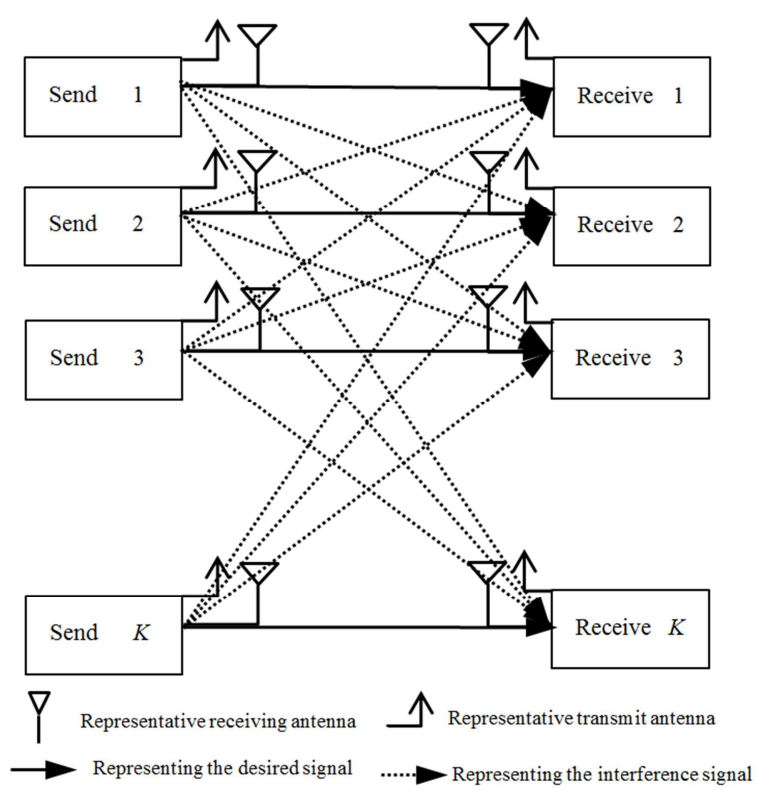

Figure 1. Multi-user system interference model.
Considering the zero-forcing of the received signal by the interference-forcing matrix in the receiver, the signal after zero-forcing can be expressed as

$$
\tilde{\boldsymbol{s}}_{i}=\boldsymbol{U}_{i}^{\mathrm{H}} \boldsymbol{y}_{i}=\boldsymbol{U}_{i}^{\mathrm{H}} \boldsymbol{H}_{i, i} \boldsymbol{V}_{i} \boldsymbol{s}_{i}+\boldsymbol{U}_{i}^{\mathrm{H}} \sum_{j=1, j \neq i}^{K} \boldsymbol{H}_{i, j} \boldsymbol{V}_{j} \boldsymbol{s}_{j}+\boldsymbol{U}_{i}^{\mathrm{H}} \boldsymbol{n}_{i}
$$

$\boldsymbol{U}_{i}$ is specified as the interference-forcing matrix (combiner), so the ideal interference alignment condition can be inferred to be

$$
\begin{gathered}
\operatorname{rank}\left(\boldsymbol{U}_{i}^{\mathrm{H}} \boldsymbol{H}_{i, i} \boldsymbol{V}_{i}\right)=d_{i} \quad \forall i \\
\boldsymbol{U}_{i}^{\mathrm{H}} \boldsymbol{H}_{i, j} \boldsymbol{V}_{j}=\mathbf{0} \quad \forall j \neq i
\end{gathered}
$$

Where equation (3) guarantees that the desired signal can be demodulated, and equation (4) guarantees that the Interference signal can be completely eliminated.

According to the LS standard, a suitable ${ }^{\boldsymbol{U}_{i}}$ can be solved according to the following formula

$$
\begin{aligned}
& \min _{\boldsymbol{U}_{i}}\left\{\left\{\left\|\boldsymbol{U}_{i}^{\mathrm{H}} \sum_{j=1}^{K} \boldsymbol{H}_{i, j} \boldsymbol{V}_{j} \boldsymbol{s}_{j}-\boldsymbol{s}_{i}\right\|_{2}\right\}\right. \\
& \triangleq \min _{\boldsymbol{U}_{i}}\left\{\operatorname{Tr}\left[\left(\boldsymbol{U}_{i}^{\mathrm{H}} \sum_{j=1}^{K} \boldsymbol{H}_{i, j} \boldsymbol{V}_{j} \boldsymbol{s}_{j}-\boldsymbol{s}_{i}\right)\left(\boldsymbol{U}_{i}^{\mathrm{H}} \sum_{j=1}^{K} \boldsymbol{H}_{i, j} \boldsymbol{V}_{j} \boldsymbol{s}_{j}-\boldsymbol{s}_{i}\right)^{\mathrm{H}}\right]\right\}
\end{aligned}
$$

Firstly, the matrix function $f_{L S}^{U}$ can be defined as follows

$$
\begin{aligned}
f_{L S}^{U} & =\left\|\boldsymbol{U}_{i}^{\mathrm{H}} \sum_{j=1}^{K} \boldsymbol{H}_{i, j} \boldsymbol{V}_{j} \boldsymbol{s}_{j}-\boldsymbol{s}_{i}\right\|_{2}^{2} \\
& =\operatorname{Tr}\left[\left(\boldsymbol{U}_{i}^{\mathrm{H}} \sum_{j=1}^{K} \boldsymbol{H}_{i, j} \boldsymbol{V}_{j} \boldsymbol{s}_{j}-\boldsymbol{s}_{i}\right)\left(\boldsymbol{U}_{i}^{\mathrm{H}} \sum_{l=1}^{K} \boldsymbol{H}_{i, l} \boldsymbol{V}_{l} \boldsymbol{s}_{l}-\boldsymbol{s}_{i}\right)^{\mathrm{H}}\right] \\
& =\operatorname{Tr}\left[\begin{array}{l}
\boldsymbol{U}_{i}^{\mathrm{H}}\left[\sum_{j=1}^{K} \sum_{l=1}^{K} \boldsymbol{H}_{i, j} \boldsymbol{V}_{j} \boldsymbol{s}_{j} \boldsymbol{s}_{l}^{\mathrm{H}} \boldsymbol{V}_{l}^{\mathrm{H}} \boldsymbol{H}_{i, l}^{\mathrm{H}}\right] \boldsymbol{U}_{i} \\
-\boldsymbol{U}_{i}^{\mathrm{H}} \sum_{j=1}^{K} \boldsymbol{H}_{i, j} \boldsymbol{V}_{j} \boldsymbol{s}_{j} \boldsymbol{s}_{i}^{\mathrm{H}}-\sum_{l=1}^{K} \boldsymbol{s}_{i}^{\mathrm{H}} \boldsymbol{S}_{l}^{\mathrm{H}} \boldsymbol{V}_{l}^{\mathrm{H}} \boldsymbol{H}_{i, l}^{\mathrm{H}} \boldsymbol{U}_{i}+\boldsymbol{s}_{i} \boldsymbol{s}_{i}^{\mathrm{H}}
\end{array}\right]
\end{aligned}
$$

Then, we can know $\frac{\partial \operatorname{Tr}\left[\boldsymbol{A} \boldsymbol{U}_{k}\right]}{\boldsymbol{U}_{k}}=\frac{\partial \operatorname{Tr}\left[\boldsymbol{U}_{k} \boldsymbol{A}\right]}{\boldsymbol{U}_{k}}=\boldsymbol{A}$, so we can get the follow formula [8]

$$
\frac{\partial f_{L S}^{U}}{\boldsymbol{U}_{i}}=\boldsymbol{U}_{i}^{\mathrm{H}}\left[\sum_{i=1}^{K} \sum_{j=1}^{K} \boldsymbol{H}_{i, j} \boldsymbol{V}_{j} \boldsymbol{s}_{j} \boldsymbol{s}_{l}^{\mathrm{H}} \boldsymbol{V}_{l}^{\mathrm{H}} \boldsymbol{H}_{i, l}^{\mathrm{H}}\right]-\sum_{l=1}^{K} \boldsymbol{s}_{i} \boldsymbol{s}_{l}^{\mathrm{H}} \boldsymbol{V}_{l}^{\mathrm{H}} \boldsymbol{H}_{i, t}^{\mathrm{H}}
$$

The best $\boldsymbol{U}_{i}$ is obtained, when $\frac{\partial f_{L S}^{U}}{\boldsymbol{U}_{i}}=0$ is satisfied, that is,

$$
\begin{aligned}
& \boldsymbol{U}_{i}^{\mathrm{H}}\left[\sum_{j=1}^{K} \sum_{l=1}^{K} \boldsymbol{H}_{i, j} \boldsymbol{V}_{j} \boldsymbol{s}_{j} \boldsymbol{s}_{l}^{\mathrm{H}} \boldsymbol{V}_{l}^{\mathrm{H}} \boldsymbol{H}_{i, l}^{\mathrm{H}}\right]=\sum_{l=1}^{K} \boldsymbol{s}_{i} \boldsymbol{s}_{l}^{\mathrm{H}} \boldsymbol{V}_{l}^{\mathrm{H}} \boldsymbol{H}_{i, t}^{\mathrm{H}} \\
& \boldsymbol{U}_{i}^{\mathrm{H}}=\left[\sum_{l=1}^{K} \boldsymbol{s}_{i} \boldsymbol{s}_{l}^{\mathrm{H}} \boldsymbol{V}_{l}^{\mathrm{H}} \boldsymbol{H}_{i, t}^{\mathrm{H}}\right]\left[\sum_{j=1}^{K} \sum_{l=1}^{K} \boldsymbol{H}_{i, j} \boldsymbol{V}_{j} \boldsymbol{s}_{j} \boldsymbol{s}_{l}^{\mathrm{H}} \boldsymbol{V}_{l}^{\mathrm{H}} \boldsymbol{H}_{i, t}^{\mathrm{H}}\right]^{-1} \\
& \boldsymbol{U}_{i}=\left[\left[\sum_{l=1}^{K} \boldsymbol{s}_{i} \boldsymbol{s}_{l}^{\mathrm{H}} \boldsymbol{V}_{l}^{\mathrm{H}} \boldsymbol{H}_{i, j}^{\mathrm{H}}\right]\left[\sum_{j=1}^{K} \sum_{l=1}^{K} \boldsymbol{H}_{i, j} \boldsymbol{V}_{j} \boldsymbol{s}_{j} \boldsymbol{s}_{l}^{\mathrm{H}} \boldsymbol{V}_{l}^{\mathrm{H}} \boldsymbol{H}_{i, t}^{\mathrm{H}}\right]^{-1}\right]^{\mathrm{H}} \\
& =\left[\sum_{j=1}^{K} \sum_{l=1}^{K} \boldsymbol{H}_{i, l} \boldsymbol{V}_{l} \boldsymbol{s}_{l} \boldsymbol{s}_{j}^{\mathrm{H}} \boldsymbol{V}_{j}^{\mathrm{H}} \boldsymbol{H}_{i, j}^{\mathrm{H}}\right]^{-1}\left[\sum_{l=1}^{K} \boldsymbol{H}_{i, l} \boldsymbol{V}_{l} \boldsymbol{s}_{l} \boldsymbol{s}_{i}^{\mathrm{H}}\right]
\end{aligned}
$$

Next, $\boldsymbol{V}_{i}$ is designed in the reverse link. Since the channels are opposite, that is, the opposite channel satisfies $\overline{\boldsymbol{H}}_{i, l}=\boldsymbol{H}_{i, l}^{\mathrm{H}}$, by exchanging $\boldsymbol{U}_{i}$ and $\boldsymbol{V}_{i}$ as the precoding matrix and the zeroforcing matrix, the optimal precoding matrix $V_{i}$ can be obtained as follow 


$$
\boldsymbol{V}_{i}=\left[\sum_{j=1}^{K} \sum_{l=1}^{K} \overline{\boldsymbol{H}}_{i,,} \boldsymbol{U}_{l} \boldsymbol{s}_{l} \boldsymbol{s}_{j}^{\mathrm{H}} \boldsymbol{U}_{j}^{\mathrm{H}} \overline{\boldsymbol{H}}_{i, j}^{\mathrm{H}}\right]^{-1}\left[\sum_{l=1}^{K} \overline{\boldsymbol{H}}_{i, l} \boldsymbol{U}_{l} \boldsymbol{s}_{l} \boldsymbol{s}_{i}^{\mathrm{H}}\right]
$$

\section{Algorithm Description}

\subsection{LS-IA Algorithm}

The traditional LS considers that the design of the transceiver in the iterative calculation should not be related to the data stream $[4,8,10]$. In order to eliminate the influence of the data stream, the mathematically expected processing is performed on the data stream $\boldsymbol{s}_{j}$ in the calculation of $\boldsymbol{U}_{i}$ and $\boldsymbol{V}_{i}$. In general, the symbol stream satisfies the following formula

$$
\mathrm{E}\left(\boldsymbol{s}_{j} \boldsymbol{s}_{j}^{\mathrm{H}}\right)=P \boldsymbol{I}, \quad \mathrm{E}\left(\boldsymbol{s}_{i} \boldsymbol{s}_{j}^{\mathrm{H}}\right)=\boldsymbol{0}, i \neq j
$$

So we can get the best $\boldsymbol{U}_{i}$ and $\boldsymbol{V}_{i}$ of formula (11-12)

$$
\begin{aligned}
\boldsymbol{U}_{i} & =\left[\sum_{j=1}^{K} \boldsymbol{H}_{i, j} \boldsymbol{V}_{j} \boldsymbol{V}_{j}^{\mathrm{H}} \boldsymbol{H}_{i, j}^{\mathrm{H}}\right]^{-1} \boldsymbol{H}_{i, i} \boldsymbol{V}_{i} \\
\boldsymbol{V}_{i} & =\left[\sum_{j=1}^{K} \overline{\boldsymbol{H}}_{i, j} \boldsymbol{U}_{j} \boldsymbol{U}_{j}^{\mathrm{H}} \overline{\boldsymbol{H}}_{i, j}^{\mathrm{H}}\right]^{-1} \overline{\boldsymbol{H}}_{i, i} \boldsymbol{U}_{i}
\end{aligned}
$$

In order to satisfy the ideal interference alignment condition of formula (3-4) in the traditional method, $V_{i}$ is firstly initialized to a random unitary matrix, and then equation (11) and formula (12) are iterated through the loop until the set iteration number is completed or some conditions are met. Finally,the data stream is detected passes through $\boldsymbol{U}_{i}$ and $\boldsymbol{V}_{i}$, where $\boldsymbol{U}_{i}$ and $\boldsymbol{V}_{i}$ is obtained by the above steps. The definition algorithm is summarized as follows, where orth represents orthogonalization and $\boldsymbol{U}_{i} \Leftarrow \operatorname{orth}\left\{\boldsymbol{U}_{i}\right\}$ represents orthogonalization of $\boldsymbol{U}_{i}$, the same applies hereinafter.

\section{Table 1. LS-IA algorithm.}

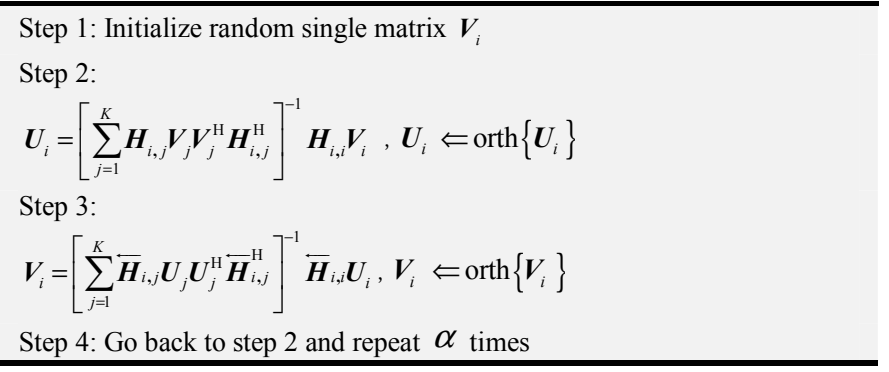

\subsection{SDA-LS-IA Algorithm}

In fact, the result of the formula (11-12) of the conventional method is the statistical result of the formula (89), which is expected to be superimposed on the data stream $\boldsymbol{s}_{j}$ of the formula (8-9)[13]. So the two are not strictly equal, especially if the data traffic is not large enough. This paper proposes an improved algorithm by making $\boldsymbol{s}_{j}=\tilde{\boldsymbol{s}}_{j}$ as follows:
First, the precoding matrices $\boldsymbol{U}_{i}$ and $\boldsymbol{V}_{i}$ are iteratively calculated using the traditional LS algorithm; then, the symbol detection is performed; finally, the iterative operation is performed again according to $\boldsymbol{s}_{j}=\tilde{\boldsymbol{s}}_{j}$, and $\boldsymbol{U}_{i}$ and $\boldsymbol{V}_{i}$ are calculated.

So the best $U_{i}$ and $V_{i}$ can be obtained as follows

$$
\begin{gathered}
\boldsymbol{U}_{i}=\left[\sum_{j=1}^{K} \sum_{l=1}^{K} \boldsymbol{H}_{i, l} \boldsymbol{V}_{l} \tilde{\boldsymbol{s}}_{l} \tilde{\boldsymbol{s}}_{j}^{\mathrm{H}} \boldsymbol{V}_{j}^{\mathrm{H}} \boldsymbol{H}_{i, j}^{\mathrm{H}}\right]^{-1}\left[\sum_{l=1}^{K} \boldsymbol{H}_{i, l} \boldsymbol{V}_{l} \tilde{\boldsymbol{s}}_{l} \tilde{\boldsymbol{s}}_{i}^{\mathrm{H}}\right] \\
\boldsymbol{V}_{i}=\left[\sum_{j=1}^{K} \sum_{l=1}^{K} \overline{\boldsymbol{H}}_{i, \boldsymbol{U}} \boldsymbol{U}_{l} \tilde{\boldsymbol{s}}_{l} \tilde{\boldsymbol{s}}_{j}^{\mathrm{H}} \boldsymbol{U}_{j}^{\mathrm{H}} \overline{\boldsymbol{H}}_{i, j}^{\mathrm{H}}\right]^{-1}\left[\sum_{l=1}^{K} \overline{\boldsymbol{H}}_{i, l} \boldsymbol{U}_{l} \tilde{\boldsymbol{s}}_{l} \tilde{\boldsymbol{s}}_{i}^{\mathrm{H}}\right]
\end{gathered}
$$

Defining $\alpha$ and $\beta$ as iteration values. The algorithm is summarized as follows:

\section{Table 2. SDA-LS-IA algorithm.}

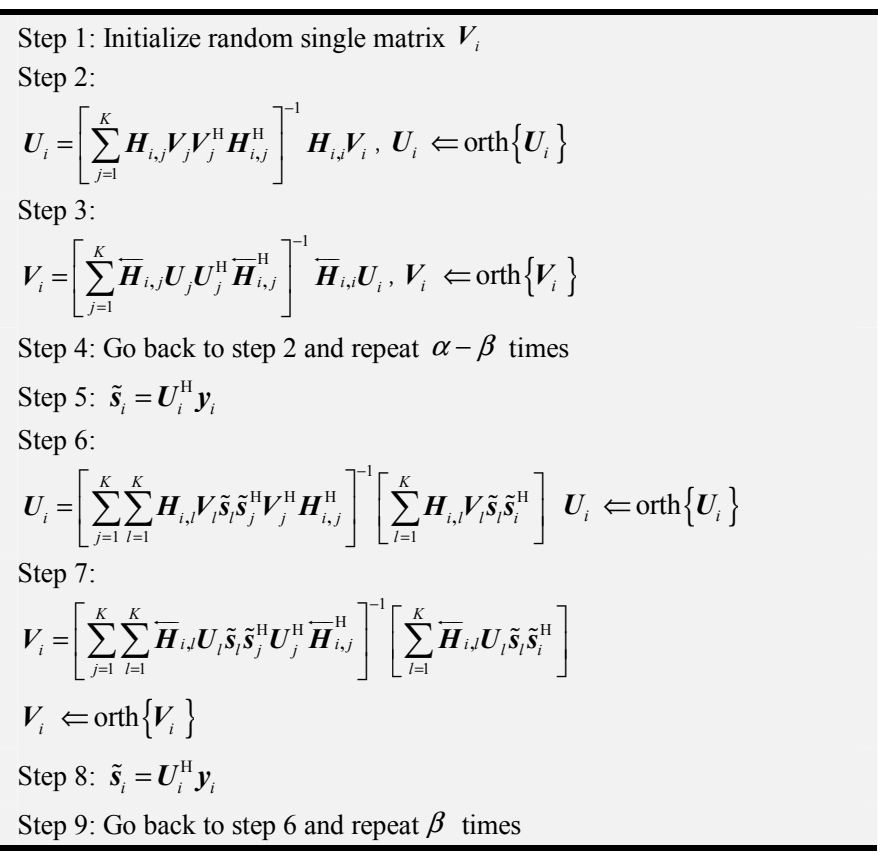

\section{Simulation Results and Analysis}

The performance of the proposed SDA-LS algorithm is simulated by computer, and the corresponding simulation parameters are set according to Table 3 . The total number of iterations is set to 70 , that is, $\alpha=70$.

Table 3. Simulation parameter settings.

\begin{tabular}{ll}
\hline The total number of iterations $\alpha$ & 70 \\
User number $K$ & 3 \\
Transmitting antenna $N$ & 4 \\
Receiving antenna $M$ & 4 \\
Data stream $d$ & 2 \\
Channel traversal times & 100000 \\
Modulation Mode & QPSK \\
\hline
\end{tabular}

According to the algorithm summarized in Table 2, the set $\beta$ is to optimize the performance of the algorithm. When $\beta$ is too small, the gain obtained from equation (13- 
14) (that is, SDA-LS-IA algorithm) is small or even no gain; when $\beta$ is too large, due to the iterations number of equation (11-12) (that is, LS-IA algorithm ) is small, and the matrices $\boldsymbol{U}_{i}$ and $\boldsymbol{V}_{i}$ do not converge, resulting in the signal detected by equation (2) being substantially mismatched with the actual transmitted signal, that is to say, the mean square error (MSE) and the bit error rate (BER) are large.

So the best $\beta$ should be obtained by some special means and should change as the wireless parameters change.

Figures 2 and 3 are obtained with the SNR setting of $30 \mathrm{~dB}$. Figure 2 shows the simulation results of the bit error rate (BER) under different $\beta$. Figure 3 shows the simulation results of the mean square error (MSE) at different $\beta$. It can be seen from Figure 2 that the BER reaches the lowest at $\beta=8$; from Figure 3, it can be concluded that the MSE is also the lowest at $\beta=8$. Therefore, combining the two graphs shows that the performance of the algorithm is optimal when $\beta=8$.

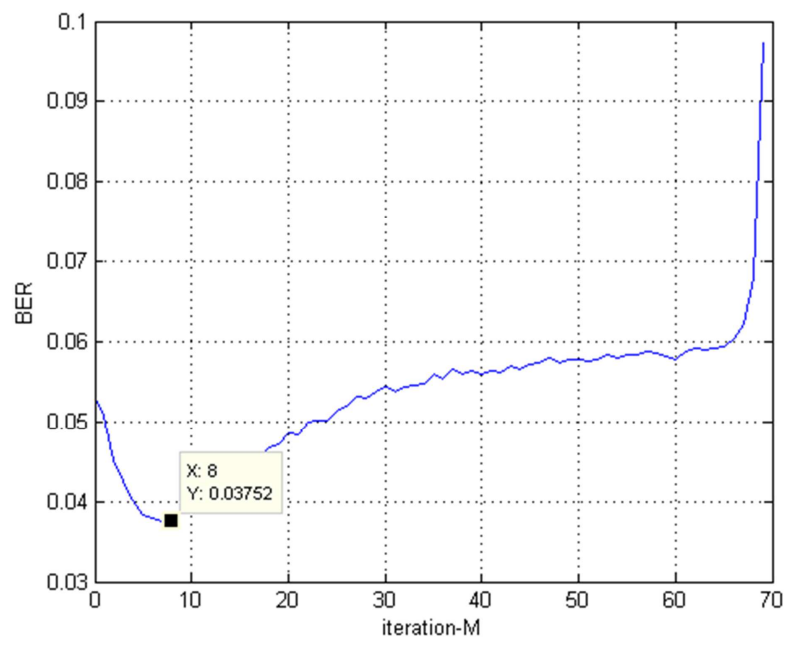

Figure 2. BER simulation results under different $\beta$.

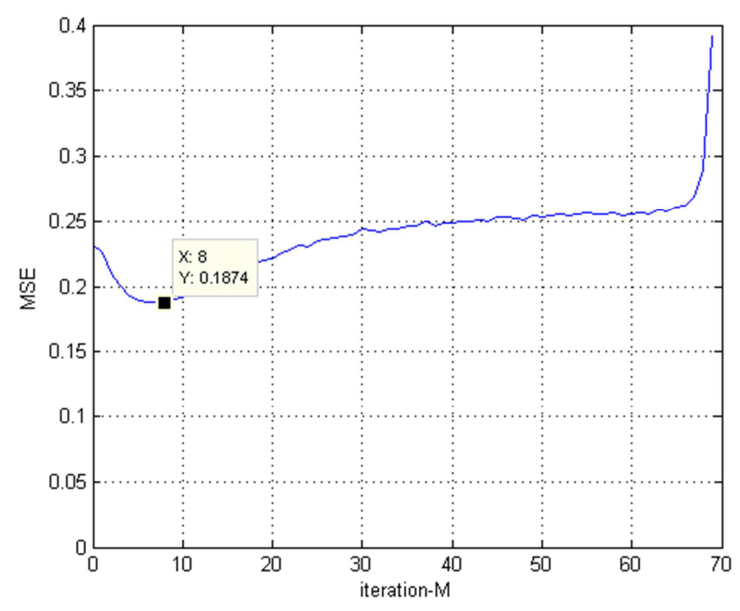

Figure 3. MSE simulation results under different $\beta$.

In accordance with Table 3, the relevant parameters are set, and Figure 4 and 5 are obtained through simulation. The horizontal axis of Figure 4 represents the variance of the algorithm, and the vertical axis of Figure 4 represents the probability density function (PDF) of MSE. It can be seen from of Figure 4 that the simulation curve of the proposed algorithm is obviously left, that is, the probability of SDA-LS-IA is larger in the region with lower variance. Therefore, it can be concluded that SDALS-IA has significantly better MSE probability density performance than traditional LS-IA in the case where the precoder initialization matrix is the same.

In addition, analysis of Figure 5 can lead to the conclusion that SDA-LS-IA exhibits consistent performance with LS-IA when the number of iterations is small; with the increasing of the number of iterations, SDA-LS-IA exhibits better MSE performance than traditional LS-IA.

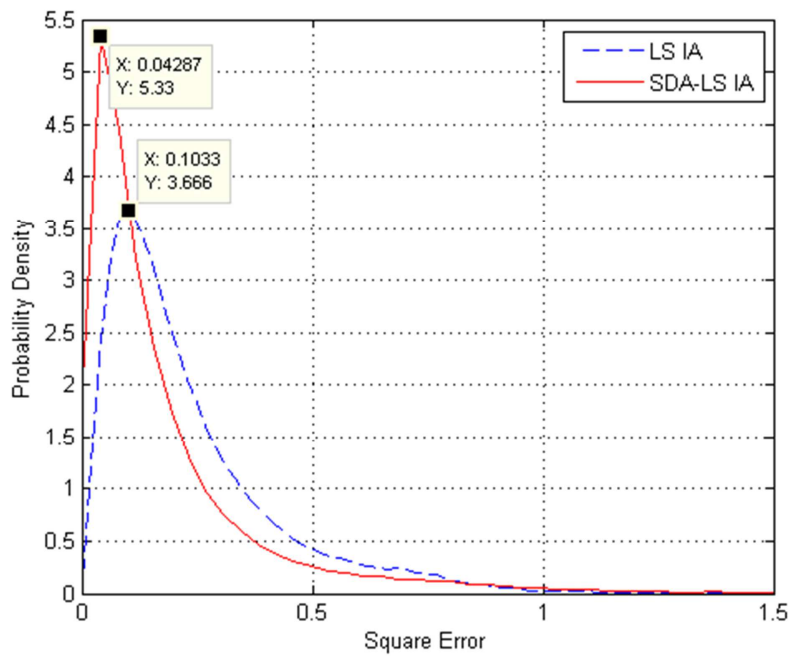

Figure 4. Probability density function of MSE.

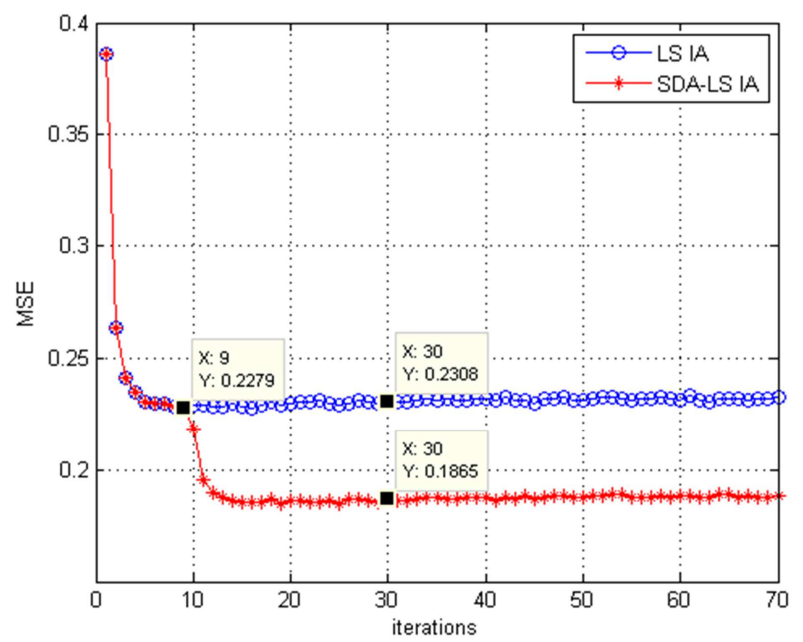

Figure 5. MSE comparison chart of SDA-LA-IA and LS-IA.

Figure 6 depicts the BER curves for the iterative calculation of traditional LS-IA and SDA-LS-IA under different SNR conditions. From Figure 6, we can conclude that SDA-LS-IA can also exhibit the same performance as traditional LS-IA in areas with low SNR; BER performance obtained by SDA-LS-IA as SNR increases apparently rising and gradually becoming stable, the gain of SDA-LS-IA can reach more than $20 \mathrm{~dB}$ by comparison to the traditional LS. 


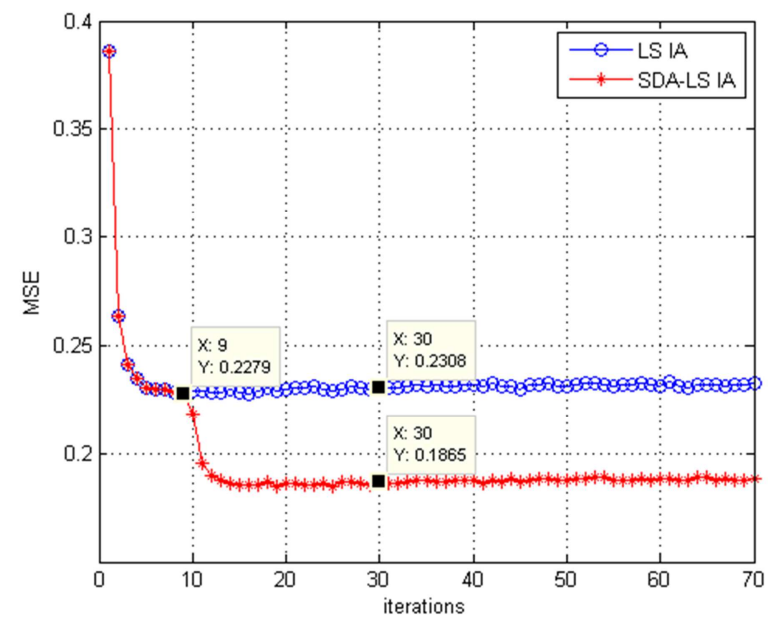

Figure 6. BER comparison of SDA-LS-IA and LS-IA.

\section{Conclusion}

Aiming at the shortcomings of traditional LS-IA algorithm in the transceiver design, this paper proposes a SDA-LS-IA iterative algorithm based on symbol detection assistance. From Figure 4, 5, and 6, we can see that as the number of iterations increases, the performance of both algorithms tends to be stable. SDA-LS-IA has better BER, MSE and PDF than LS-IA performance. So, the following conclusions can be drawn. Since the algorithm based on symbol detection is introduced on the basis of the traditional algorithm, the proposed detection algorithm shows better MSE and BER performance than the traditional algorithm.

Compared with LS-IA, SDA-LS-IA increases the number of iterations, which improves the performance of IA. In theory, performance improvement is large and the complexity is not improved much. Therefore, the proposed algorithm has research significance. The disadvantage of this paper is that there is no complexity analysis of the two algorithms.

\section{Acknowledgements}

This research is supported by the Key Laboratory of Wireless Sensor Network \& Communication, Shanghai Institute of Microsystem and Information Technology, Chinese Academy of Sciences (grant 2016002).

\section{References}

[1] Anming Dong, Haixia Zhang, Dongfeng Yuan, Xiaotian Zhou. 2016. Interference Alignment Transceiver Design by Minimizing the Maximum Mean Square Error for MIMO Interfering Broadcast Channel. IEEE Transaction on Vehicular Technology. 65, 8 (August. 2016), 3024-6036.
[2] Galymzhan Nauryzbayev, Emad Alsusa. 2016. Enhanced Multiplexing Gain Using Interference Alignment Cancellation in Multi-cell MIMO Networks. IEEE Transaction on Information Technology. 62, 1 (January.2016), 357-369.

[3] Zhiyu Cheng, Natasha Devroye, Tang Liu. 2016. The Degrees of Freedom of Full-Duplex Bidirectional Interference Networks With and Without a MIMO Relay. IEEE Transaction on wireless Communications. 15, 4 (April.2016), 2912-2924.

[4] Krishna Gomadam, Viveck R. Cadambe, Syed A. Jafar. 2011. A Distributed Numerical Approach to Interference Alignment and Applications to Wireless Interference Networks. IEEE Transaction on Information Theory. 57, 6 (June. 2011), 33093322.

[5] Suh, C. and Tse, D. 2008. Interference Alignment for Cellular Networks [C]. 46th Annual Allerton Conference on Communications, Control and Computing (Illinois, USA, Sep. 2008).

[6] Vasilis N, Mohammad A, and Giuseppe C. Cellular Interference Alignment. IEEE Trans. Inf. Theory. 2015, 61(3), 1194-1217.

[7] K. Gomadam, V. R. Cadambe, and S. A. Jafar. A distributed numerical approach to interference alignment and applications to wireless interference networks. IEEE Trans. Inf. Theory. 57, 6 (Jun. 2011), 3309-3322.

[8] Razavi S M, and Ratnarajah T. Adaptive LS and MMSE based beamformer design for multiuser MIMO interference channels. IEEE Trans. Veh. Technol. 65, 1(2016), 132-144.

[9] Hakjea Sung, Seok-Hwan, kyoung-Jae Lee. 2010. Linear Precoder Designs for K-user Interference Channels. IEEE Transactions on Wireless Communications. 9, 1(January, 2010), 291-300.

[10] Paula Aquilina, Tharmalingam Ratnarajah. 2015. Performance Analysis of IA Techniques in the MIMO IBC With Imperfect CSI.IEEE Transaction on Communications. 63, 4 (April.2015), 1259-1268.

[11] Leonard H. Grokop, David N. C. Tse, Roy D. Yates. 2011.Interference Alignment for Line-of-sight Channels. IEEE Transactions on Information Theory. 57, 9 (September, 2011), 5820-5832.

[12] Cheuk Ting Li, Ayfer Ozgur.2016. Channel Diversity Needed for Vector Space Interference Alignment. IEEE Transactions on Information Theory. 62, 4 (April, 2016), 1942-1956.

[13] Jia, G. Q., Pan, Y., Du, J. J., and Ji, X. H. 2018. Symbol detection aided minimum mean square error interference alignment. In 2018 IEEE MTT-S International Wireless Symposium. (The Chengdu, The China, May 06-09, 2018). 